\title{
Consumo y digestibilidad en novillos cebuínos en un sistema silvopastoril intensivo
}

\author{
Gaviria-Uribe, X. ${ }^{@} ;$ Naranjo-Ramírez, J.F.' $;$ Bolívar-Vergara, D.M. ${ }^{1}$ y Barahona-Rosales, R. ${ }^{1}$
}

'Departamento de Producción Animal. Facultad de Ciencias Agrarias. Universidad Nacional de Colombia. Sede Medellín. Colombia. ${ }^{2}$ Facultad de Medicina Veterinaria y Zootecnia. Grupo INCA-CES. Universidad CES. Medellín. Colombia.

\section{Palabras ClaVe adicionales}

Ganadería tropical.

Leucaena leucocephala.

Nutrientes en heces.

\section{RESUMEN}

La determinación del consumo voluntario de materia seca (CMS) por los animales, es indispensable para determinar su capacidad productiva y su estado nutricional. Existen muchas técnicas para la estimación del CMS, siendo una de ellas la técnica de n-alcanos la cual permite además estimar la selectividad de los forrajes consumidos. El presente trabajo tuvo como objetivo estimar el consumo voluntario y la excreción fecal de nutrientes por novillos cebuínos alimentados en un sistema silvopastoril intensivo (SSPi). El estudio se realizó en el Centro Experimental Cotové, propiedad de la Universidad Nacional de Colombia. Se evaluaron seis novillos castrados con un peso promedio de $381 \pm 12 \mathrm{~kg}$ y se utilizó la técnica de alcanos para determinar CMS. El CMS promedio de forraje fue de $9,54 \mathrm{~kg} \mathrm{dia}^{-1}$, del cual, el 75,24 \% correspondió a consumo de gramíneas y el $24,76 \%$ a consumo de leguminosa. En promedio, el consumo de nutrientes $(\mathrm{kg})$ fue de 1,$33 ; 5,8 ; 4,02 ; 1,13 ; 0,058$ y 0,021 para PC, FDN, FDA, Cenizas, Ca y $\mathrm{P}$ respectivamente. Estos consumos fueron adecuados para el tipo de animales del estudio aportando una dieta de buena calidad nutricional. La digestibilidad fue del $53 \%$ para el tratamiento sin inclusión del suplemento y del $58 \%$ para el tratamiento con suplemento, lo que junto con el CMS, permite afirmar que los animales satisficieron sus requerimientos de los principales nutrientes. Las cantidades excretadas $(\mathrm{kg})$ fueron 4,$15 ; 0,44 ; 2,56 ; 1,78 ; 0,8 ; 0,054$ y 0,02 para MS, PC, FDN, FDA, Cenizas, Ca y $P$, respectivamente. La excreción fecal de $N$ por animal al año se calculó en $29,9 \mathrm{~kg}$, contribuyendo a la producción de biomasa de las gramíneas acompañantes en el SSPi.

\section{Intake and digestibility of nutrients in Zebu steers grazing in intensive silvopastoral system}

\section{SUMMARY}

\section{ADDITIONAL KEYWORDS \\ Tropical beef production. Leucaena leucocephala. Fecal nutrients.}

\section{INFORMACIÓN}

\section{Cronología del artículo.}

Recibido/Received: 16.1.2014

Aceptado/Accepted: 12.1.2015

On-line: 16.3.2015

Correspondencia a los autores/Contact e-mail:

xgavirio@unal.edu.co
The determination of the dry matter intake (DMI) of animals is essential to determine the productive ability and nutritional status of domestic animals. Many techniques exist for estimating DMl and that of n-alkanes has shown special appeal, since it also allows the determination of the selectivity of the fodder consumed. The present study aimed to estimate voluntary feed intake and fecal nutrient excretion in Zebu steers fed in an intensive silvopastoral system (ISS). The study was conducted at the Cotové Research Center of the National University of Colombia in Santa Fe, Antioquia. Six steers with an average weight of $381.16 \pm 12.1 \mathrm{~kg}$ were evaluated and the n-alkane technique was used to determine DMI. The average forage DMI intake was $9.54 \mathrm{~kg}$ day ${ }^{1}$ of which, $75.24 \%$ corresponded to intake of grasses and $24.76 \%$ to legume intake. Nutrient intake $(\mathrm{kg})$ was $1.33,5.8,4.02,1.13,0.058$ and 0.021 for CP, NDF, ADF, ashes, Ca and P, respectively. These intakes were adequate for the type of animals evaluated and provided a diet of good nutritional quality. The digestibility was $53 \%$ for the diet without supplementation and $58 \%$ for the diet with supplementation, which together with the DMI data, suggests that animals met their requirements of key nutrients. In turn, nutrient excretion $(\mathrm{kg})$ was $4.15,0.44,2.56,1.78,0.8,0.054$ y 0.02 for DN, $C P, N D F, A D F$, ashes, $C a$ and $P$, respectively. Faecal $N$ excretion was calculated at 29,9 $\mathrm{kg}$ per animal per year, and this contributes to the biomass production of the ISS grasses.

\section{INTRODUCCIÓN}

La producción ganadera es considerada una actividad de alto impacto sobre el medio ambiente. Por ejemplo, la mayor parte del nitrógeno consumido por los animales es excretado en heces y orina y muy poco de este elemento es retenido en productos animales como carne y leche (Herrero et al., 2013). Bajo condiciones adecuadas de manejo, las heces y orina se convierten en una vía importante en el reciclaje de nutrientes como nitrógeno y fósforo al suelo y se ha demostrado que la cantidad de nutrientes que retornan por esta vía cambia en función del tipo de animal, su estado fisiológico y la concentración de nutrientes en la dieta consumida (Crespo et al., 2000).

No solo porque está asociado con la excreción de nutrientes, sino porque da una idea de la capacidad productiva y del estado nutricional de los animales (Dove et al., 2000), una de las características importantes a evaluar en los sistemas de producción es el consumo. Sin embargo, este es un parámetro de difícil medición, especialmente bajo condiciones de pastoreo. Una técnica de gran utilidad para estimar el consumo 
de forrajes por animales en pastoreo es la utilización de alcanos como marcadores internos (Dove y Mayes, 1991; Gedir y Hudson, 2000; Giráldez et al., 2006; Ferreira et al., 2007). Los alcanos son cadenas de hidrocarburos saturados de 21 a 37 átomos de carbono presentes en la cera de la mayoría de las plantas, siendo el $\mathrm{C}_{29}$ $\mathrm{C}_{31}$ y $_{33^{\prime}}$ los más predominantes en la mayoría de las especies de pastos. Además de ser útiles para estimar el consumo de materia seca de animales en diferentes condiciones de pastoreo, los alcanos también posibilitan estimar la composición de la dieta consumida (Dove y Mayes, 1991).

En los últimos años ha aumentado el interés por convertir los sistemas ganaderos en sistemas cada vez más intensivos, que permitan alcanzar mayor productividad, junto con mayor competitividad y sostenibilidad. Sistemas de producción como los sistemas silvopastoriles intensivos (SSPi) han mostrado ser una buena opción para lograr una ganadería sostenible. Estos sistemas se caracterizan por tener altas densidades (más de 10000 por hectárea) de arbustos forrajeros como la acacia forrajera (Leucaena leucocephala) asociados a pastos mejorados de alta producción de biomasa y árboles introducidos bajo modelos de pastoreo rotacional intensivos (Murgueitio et al., 2011; Cuartas et al., 2014).

A pesar de su promesa (Cuartas et al., 2014), existen pocos estudios en los que se haya determinado el consumo de materia seca y de nutrientes y la selectividad forrajera en SSPi por novillos cebuinos, los cuales conforman la gran mayoría de los animales para producción de carne en América tropical. Este conocimiento es de vital importancia para determinar cuáles son las limitaciones nutricionales de estos sistemas y proponer posibles estrategias de suplementación a fin de incrementar la productividad de estos sistemas, la cual es baja. El objetivo de este estudio fue evaluar la selectividad, el consumo y la digestibilidad de forrajes de bovinos de carne pastoreando en sistemas silvopastoriles intensivos con diferentes forrajes.

\section{MATERIAL Y MÉTODOS}

\section{LOCALIZACIÓN}

El estudio tuvo lugar en el Centro Experimental Cotové, propiedad de la Universidad Nacional de Colombia sede Medellín, ubicado en el municipio de Santafé de Antioquia a 74 kilómetros de distancia de Medellín. Su zona de vida según la clasificación ecológica de Holdridge es bosque seco tropical (bs-T) y está a una altura de $540 \mathrm{msnm}$, con una temperatura promedio de $27^{\circ} \mathrm{C}$ y una precipitación de $1100 \mathrm{~mm}$ año-1 .

\section{DESCRIPCIÓN DEL SISTEMA DE PRODUCCIÓN}

El SSPi utilizado tenía un área total de 5,6 ha y estaba compuesto por arbustos de leucaena (Leucaena leucocephala) sembrados en una densidad de 10000 arbustos ha-1 asociados a pasto guinea (Megathyrsus maximus Jacq) variedad tanzania y pasto estrella (Cynodon plectostachyus). El pasto estrella constituía menos de $3 \%$ de la biomasa total disponible a partir de las praderas. El pastoreo en dicho sistema se realizó mediante rotación en franjas de $1200 \mathrm{~m}^{2}$, con un día de ocupación y 44 días de descanso para un periodo de rotación total de 45 días.

\section{MANEJO ALIMENTICIO DE LOS ANIMALES}

En el SSPi pastorearon 20 animales de raza cebú comercial, castrados y con una edad promedio de 3 años. Estos animales consumieron a voluntad los forrajes del SSPi: leucaena, pasto guinea variedad tanzania y pasto estrella, siendo el consumo de estrella muy limitado debido a su poca presencia en el potrero. Además, tuvieron acceso permanente a sal y agua a voluntad. Un grupo de diez animales recibieron $500 \mathrm{gr} \mathrm{dia}^{-1} \mathrm{de}$ un suplemento a base de harina de arroz y melaza (70 y $30 \%$, respectivamente).

\section{COMPOSICIÓN BROMATOLÓGICA DE FORRAJES Y SUPLEMENTO CONSUMIDO}

Se recolectaron muestras representativas de los forrajes consumidos por los animales en los días 1, 6 y 10 de evaluación. Dichas muestras se trasladaron al Laboratorio de Análisis Químico y Bromatológico de la Universidad Nacional, sede Medellín donde se secaron en una estufa de aire forzado a $60^{\circ} \mathrm{C}$ durante $48 \mathrm{~h}$ aproximadamente y fueron molidas a través de una malla de $1 \mathrm{~mm}$ para su posterior análisis. En una muestra del suplemento ofrecido a los animales durante todos los días de evaluación se realizaron los mismos análisis que se realizaron en los forrajes.

En las muestras de forrajes y suplemento se determinaron los contenidos de materia seca (MS) por el método ISO 6496, en estufa de aire forzado a $105{ }^{\circ} \mathrm{C}$ hasta alcanzar peso constante (ISO, 1999); proteína cruda (PC) por el método de Kjeldahl, según NTC 4657 (ICONTEC, 1999); fibra insoluble en detergente neutro y ácido (FDN y FDA, respectivamente) según técnica secuencial descrita por Van Soest et al. (1991); lignina (Van Soest et al., 1963) y grasa bruta (EE), por extracción Soxhelet por inmersión por el método NTC 668 (ICONTEC, 1973). El contenido de cenizas se determinó por incineración directa en una mufla a $500{ }^{\circ} \mathrm{C}$, según AOAC 942.05 (AOAC, 2005). El contenido de calcio y fósforo se determinó por espectrofotometría AA y U.V-VIS basado en NTC 5151 (ICONTEC, 2003) y 4981 (ICONTEC, 2001), respectivamente. Finalmente, se determinó el contenido de energía bruta por calorimetría, basado en el método ISO 9831 (ISO, 1998). Los contenidos de nutrientes estimados se muestran en la tabla I.

\section{EVALUACIÓN DEL CONSUMO Y EXCRECIÓN DE NUTRIENTES}

Las evaluaciones de consumo de forraje se realizaron en un período de diez días, utilizando la técnica de alcanos (Mayes et al., 1986). Las evaluaciones se realizaron con 6 animales escogidos al azar del grupo de 20 animales; tres de los cuales pertenecían al grupo de animales suplementados. Al momento de las mediciones, los animales tenían un peso promedio de $381 \pm 12 \mathrm{~kg}$.

Los alcanos se ofrecieron en cápsulas de gelatina con un contenido exacto de $250 \mathrm{mg}$ de una mezcla de cantidades conocidas de n-dotriacontano $\left(\mathrm{C}_{32}\right)$ y $\mathrm{n}$ hexatriacontano $\left(\mathrm{C}_{36}\right)$ (MCM Alcanos-CAPTEC). Para esto, los animales fueron primero acostumbrados a la 
Tabla I. Composición química de los forrajes ofertados en el sistema silvopastoril intensivo del Centro de Investigación Cotové (Chemical composition of forages offered in the intensive silvopastoral system at Cotové Research Center).

\begin{tabular}{lccr}
\hline \multirow{2}{*}{ Composición química } & \multicolumn{2}{c}{ Gramínea ofertada } & $\begin{array}{c}\text { Leguminosa } \\
\text { ofertada }\end{array}$ \\
\cline { 2 - 4 } & Estrella & Guinea & Leucaena \\
\hline Proteína (\%) & $10,1 \pm 2,33$ & $8,70 \pm 3,68$ & $26,1 \pm 4,74$ \\
FDN (\%) & $69,7 \pm 2,12$ & $70,1 \pm 5,02$ & $33,6 \pm 9,62$ \\
FDA (\%) & $45,0 \pm 5,16$ & $47,9 \pm 6,86$ & $29,3 \pm 10,2$ \\
Grasa bruta (\%) & $1,16 \pm 0,34$ & $1,43 \pm 0,50$ & $2,92 \pm 0,25$ \\
Valor calorífico kcal/kg & $4091 \pm 151$ & $4004 \pm 36,1$ & $4640 \pm 51,6$ \\
Cenizas (\%) & $12,3 \pm 2,28$ & $13,5 \pm 0,46$ & $8,59 \pm 1,07$ \\
Calcio (\%) & $0,37 \pm 0,05$ & $0,37 \pm 0,15$ & $1,34 \pm 0,20$ \\
Fósforo (\%) & $0,24 \pm 0,01$ & $0,20 \pm 0,02$ & $0,23 \pm 0,04$ \\
\hline
\end{tabular}

FDN= Fibra en detergente neutro; FDA= Fibra en detergente ácido; $\pm=$ Desviación típica

presencia de las personas encargadas de suministrar las cápsulas, realizando acercamientos graduales para ofrecer cápsulas vacías a los animales hasta que las consumieron sin ninguna dificultad.

Las cápsulas se administraron durante un periodo total de 12 días, con una frecuencia de dos cápsulas por día (mañana y tarde). Los primeros siete días, correspondieron al período de adaptación al marcador para lograr que la concentración de alcano liberado alcanzara un estado de equilibrio. A partir del día ocho de la administración de las cápsulas, se recolectaron muestras de heces durante cinco días consecutivos en las horas de la mañana y de la tarde. Adicionalmente, se recolectaron muestras de los forrajes consumidos en los días 1, 8 y 12 del experimento.

El contenido de alcanos se determinó tanto en los forrajes consumidos como en las heces que fueron recolectadas a partir del día 6 hasta el día 10. Para esto se generaron tres mezclas por animal, dos de ellos reuniendo las muestras de la mañana y tarde en los días 6-7 y 8-9. La tercera mezcla fue generada con muestras mañana y tarde del día 10. En el suplemento ofrecido se determinó también el contenido de alcanos. Este análisis se realizó en una sola muestra, ya que el suplemento ofrecido durante todo el periodo experimental pertenecía al mismo lote.

La extracción de los alcanos se realizó en el laboratorio de Nutrición Animal de la Universidad Nacional de Colombia-Sede Medellín, siguiendo el protocolo de Dove y Mayes (2006). Se adicionaron 0,11 g de una solución de n-docosano $\mathrm{C}_{22}$ y n-tetratriacontano $\mathrm{C}_{34}$ en n-decano (0,3 $\mathrm{mg} \mathrm{g}^{-1}$ de $\mathrm{n}$-decano) como estándar interno. Posteriormente se adicionaron 1,5 y $2 \mathrm{ml}$ de una solución de $\mathrm{KOH}$ etanólico $(1 \mathrm{M})$ a las muestras de forraje y heces, respectivamente. Las muestras fueron calentadas durante 16 horas en un horno con una temperatura de $90^{\circ} \mathrm{C}$. El extracto obtenido se disolvió utilizando heptano y agua, llevando la solución resultante de nuevo al horno hasta la evaporación total del líquido. El sólido resultante se disolvió con heptano y la solución obtenida fue filtrada a través de una columna de $5 \mathrm{~cm}$ de sílica gel. El líquido obtenido se llevó nuevamente a evaporación para finalmente disolver el extracto obtenido con 0,25 $\mathrm{ml}$ de $\mathrm{n}$-dodecano.

La cuantificación de los alcanos se realizó en el Laboratorio de Análisis Instrumental de la Universidad Nacional de Colombia, sede Medellín mediante un cromatógrafo de gases equipado con un detector de ionización de llama (FID) y una columna capilar Agilent (DB-1MS) con $250 \mu \mathrm{m}$ de diámetro interno de $30 \mathrm{~m}$. Se utilizó helio como gas de arrastre a un flujo constante de $15 \mathrm{ml} /$ minuto. Para el detector se usaron gradientes de temperatura $\left(80{ }^{\circ} \mathrm{C}\right.$ por $0,2 \mathrm{~min}, 200 \mathrm{~K} / \mathrm{min}$ hasta $\left.380{ }^{\circ} \mathrm{C}\right)$ así como para la columna $\left(200{ }^{\circ} \mathrm{C}\right.$ por $1 \mathrm{~min}, 6$ $\mathrm{K} / \mathrm{min}$ hasta $300^{\circ} \mathrm{C}$ ). La calibración del procedimiento cromatográfico se hizo con una solución que contenía una mezcla de n-alcanos $\mathrm{C}_{23}, \mathrm{C}_{25}, \mathrm{C}_{27}, \mathrm{C}_{29^{\prime}} \mathrm{C}_{31}, \mathrm{C}_{32}, \mathrm{C}_{33^{\prime}}$

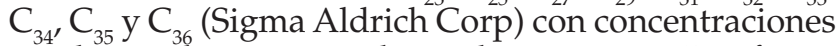
similares a las encontradas en los extractos. Los factores de respuesta se calcularon basándose en las áreas de los picos y las concentraciones conocidas de dichos estándares.

A partir de los valores de excreción diaria de $\mathrm{N}$ alcanos de los seis novillos se calculó el consumo de materia seca (CMS) usando la ecuación de Dove y Mayes (2006):

$$
\operatorname{CMS}\left(\mathrm{kg} \mathrm{dia}^{-1}\right)=\frac{\mathrm{Dj}}{\left(\frac{\mathrm{Fj}}{\mathrm{Fi}}\right)(\mathrm{Hi}-\mathrm{Hj})}
$$

Dónde:

$\mathrm{Fi}=$ concentración $\left(\mathrm{mg} \mathrm{kg}{ }^{-1}\right.$ de MS) del n-alcano de cadena impar en las heces;

$\mathrm{Fj}=$ concentración (mg kg-1 de MS) del n-alcano sintético de cadena par $\left(\mathrm{C}_{32} \mathrm{o} \mathrm{C}_{36}\right)$ en las heces;

$\mathrm{Dj}=$ cantidad del n-alcano sintético de cadena par $\left(\mathrm{C}_{32} \mathrm{o} \mathrm{C}_{36}\right)$ ofrecido $\left(\mathrm{mg} \mathrm{día}^{-1}\right)$;

$\mathrm{Hi}$ = concentración ( $\mathrm{mg} \mathrm{kg}^{-1}$ de MS) del n-alcano de cadena impar natural del forraje;

$\mathrm{Hj}=$ concentración ( $\mathrm{mg} \mathrm{kg}{ }^{-1}$ de MS) del n-alcano sintético de cadena par $\left(\mathrm{C}_{32} \mathrm{o} \mathrm{C}_{36}\right)$ en el forraje.

Las concentraciones de $\mathrm{Hi}$ y $\mathrm{Hj}$ se calcularon en base a las estimaciones de composición de la dieta. Los estimados de consumos mostrados son el promedio de los estimados obtenidos al usar los ratios $\mathrm{C}_{32}: \mathrm{C}_{31^{\prime}}$

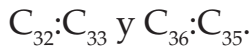

Para determinar la digestibilidad de la materia seca se usó la proporción entre la tasa de liberación de $\mathrm{C}_{36} \mathrm{y}$ la concentración de dicho marcador en las heces $\left(\mathrm{H}_{36}\right)$, usando la siguiente ecuación de Dove y Mayes (2006):

Digestibilidad $=\left(\frac{\text { Consumo }\left(\mathrm{kg} \mathrm{dia} \mathrm{a}^{-1}\right)-\text { Excreción fecal }\left(\mathrm{kg} \mathrm{dia} \mathrm{a}^{-1}\right)}{\text { Consumo }(\mathrm{kg} \text { día }}\right)$

\section{PRODUCCIÓN Y COMPOSICIÓN DE LAS HECES}

Las muestras de heces recolectadas a partir del día 8 hasta el día 12, en la mañana y en la tarde, fueron congeladas y posteriormente se trasladaron al Laboratorio de Análisis Químico y Bromatológico de la Universidad Nacional, sede Medellín donde se secaron en una estufa de ventilación forzada a $60{ }^{\circ} \mathrm{C}$ y luego fueron molidas a través de una malla de $1 \mathrm{~mm}$ para su 
posterior análisis. Para estas muestras se determinaron los contenidos de: proteína cruda, fibra en detergente neutro, fibra en detergente ácido, cenizas, calcio y fósforo; utilizando las mismas metodologías referenciadas en el análisis de los forrajes.

Para la determinación de la producción total de heces se utilizó la siguiente ecuación:

Producción de heces $\left(\mathrm{kg} \mathrm{MS} \mathrm{día}{ }^{-1}\right)=\frac{\text { Tasa de dosis del marcador }\left(m g \text { día }{ }^{-1}\right)}{\text { Concentración fecal del marcador }\left(m g \mathrm{~kg} \mathrm{día}^{-1}\right)}$

\section{SeleCtiVIDAD DE LA DIETA}

La composición de la dieta consumida se estimó comparando el patrón de los alcanos impares $\left(\mathrm{C}_{27}\right.$ a $\mathrm{C}_{35}$ ) de los forrajes consumidos con las concentraciones de $\mathrm{N}$-alcanos, corregidas por su recuperación en las heces de animales cebú (Córdoba et al., 2008). La mejor solución entre el patrón de n-alcanos en el forraje y en las heces fue establecida por el algoritmo no negativo de mínimos cuadrados descrito por Dove y Moore (1995) que minimiza las discrepancias entre las concentraciones de cada n-alcano de cadena impar. Las muestras de heces y de forraje fueron las mismas que las descritas anteriormente. Estos métodos implican la siguiente función:

Minimizar: $\sum\left(\text { alcano calculado }_{\mathrm{i}} \text {-alcano actual }{ }_{\mathrm{i}}\right)_{\text {alcano1-n }}^{2}$

Donde:

(alcano calculado-alcano actual $\left.)_{i}\right)^{2}$ son concentraciones fecales corregidas o proporciones del total de alcanos corregidos- recuperados en heces.

La principal diferencia entre los algoritmos es el método para derivar el valor calculado de alcano fecal. A continuación se describe el enfoque utilizando dos componentes de una dieta:

\section{Alcano $_{\mathrm{i}}$ fecal calculado $=\alpha \mathrm{A}_{\mathrm{i}}+\beta \mathrm{B}_{\mathrm{i}}$}

Los valores de $\alpha$ y $\beta$ son respectivamente cantidades de componentes de la dieta A y B evaluadas como desconocidas. Las constantes $\mathrm{A}_{i} \mathrm{y}_{\mathrm{i}}$ son concentraciones corregidas de alcano fecal.

\section{ANÁLISIS ESTADÍSTICO}

Los datos obtenidos en este trabajo fueron analizados mediante un diseño completamente al azar con un arreglo de medidas repetidas en el tiempo (3 mediciones realizadas durante el período experimental), del mismo individuo (efecto aleatorio). El modelo estadístico consideró como efecto fijo el tratamiento (con y sin suplemento). Se utilizó el procedimiento MIXED del programa SAS 8.0.2, el cual tiene en cuenta efectos aleatorios y permite modelar la estructura de covarianza de los datos. Se analizaron las estructuras Simetría compuesta, Sin estructura y la Combinación de estructura autoregresiva de primer orden dentro de individuos y efecto aleatorio entre individuos (Littell et al., 1998; Littell et al., 2000).

Las estructuras de covarianza fueron comparadas utilizando el criterio de información de Akaike (AIC; Akaike, 1974) y el criterio de información bayesiano (BIC; Schwarz, 1978); valores más bajos de AIC y BIC, significan mejor ajuste del modelo. La mejor estructura de covarianza de acuerdo a los criterios de AIC y BIC fue la no estructurada.

$$
\begin{aligned}
& A I C=-2 \log L+2 p \\
& B I C=-2 \log L+p \log (N-r(X))
\end{aligned}
$$

Donde:

$p=$ número de parámetros del modelo,

$N=$ número de observaciones,

$\log L=$ valor del logaritmo natural de la función de máxima verosimilitud y

$r(X)=$ rango de la matriz $X$ (matriz de incidencia para los efectos fijos).

\section{RESULTADOS Y DISCUSIÓN}

La composición química de los forrajes ofertados (tabla I) presentó grandes contrastes en contenido de proteína y fibra entre las gramíneas y la leguminosa. En condiciones de trópico bajo es común encontrar bajos tenores de PC y altos contenidos de FDN (Barahona y Sánchez, 2005). En el presente estudio, los contenidos de FDN de la dieta total fueron disminuidos por el aporte de la leucaena, que contiene en promedio solo 33,6 \% de FDN. Esto constituye una mejora para los animales, puesto que un exceso de FDN es un limitante para aumentar el consumo y la productividad animal en el trópico.

En la tabla II se presentan los consumos de MS, especies vegetales y nutrientes, no observándose efecto de la inclusión del suplemento dada la baja cantidad ofrecida $(0,5 \mathrm{~kg}$ /animal), representando el CMS total 2,46 y $2,59 \%$ del peso vivo de los animales sin y con suplemento, respectivamente. Mediante el modelo Cornell Net Carbohydrate and Protein System (CNCPS) se estimaron consumos de MS de $8,9 \mathrm{~kg}$ día $^{-1}$ es decir un $2,34 \%$ del PV, siendo estos consumos $8 \%$ menores a los estimados mediante el uso de alcanos. Por su parte, en un SSPi compuesto por leucaena y pasto guinea, Cuartas et al. (2014) reportaron consumos promedios

Tabla II. Consumo de materia seca, especies vegetales, y nutrientes por novillos cebuínos de $380 \mathrm{~kg}$ de peso pastoreando en un SSPi basado en Leucaena leucocephala con o sin suplementación energética (Dry matter intake, plant species, and nutrients by Zebu steers $380 \mathrm{~kg}$ grazing on

\begin{tabular}{|c|c|c|c|}
\hline Consumo & Sin suplemento & Con suplemento & Valor $p$ \\
\hline MS, kg día ${ }^{-1}$ & $9,42 \pm 0,94$ & $10,1 \pm 0,58$ & 0,68 \\
\hline MS, \% del PV & $2,46 \pm 0,23$ & $2,59 \pm 0,13$ & 0,36 \\
\hline Gramínea, kg MS día-1 & $7,29 \pm 0,6$ & $7,07 \pm 0,61$ & 0,65 \\
\hline Leguminosa, kg MS día ${ }^{-1}$ & $2,13 \pm 0,55$ & $2,59 \pm 0,13$ & 0,26 \\
\hline Proteína cruda, kg día ${ }^{-1}$ & $1,24 \pm 0,17$ & $1,43 \pm 0,05$ & 0,15 \\
\hline FDN, kg día ${ }^{-1}$ & $5,81 \pm 0,52$ & $5,82 \pm 0,39$ & 0,98 \\
\hline FDA, kg día ${ }^{-1}$ & $4,01 \pm 0,37$ & $4,04 \pm 0,26$ & 0,88 \\
\hline Grasa bruta, $\mathrm{kg} \mathrm{día}^{-1}$ & $0,16 \pm 0,02$ & $0,17 \pm 0,02$ & 0,30 \\
\hline Cenizas, $\mathrm{kg} \mathrm{día}^{-1}$ & $1,12 \pm 0,10$ & $1,14 \pm 0,07$ & 0,81 \\
\hline Calcio, kg día-1 & $0,06 \pm 0,008$ & $0,06 \pm 0,001$ & 0,30 \\
\hline Fósforo, kg día ${ }^{-1}$ & $0,02 \pm 0,002$ & $0,02 \pm 0,002$ & 0,41 \\
\hline
\end{tabular}
Leucaena leucocephala SSPi with or withoutenergy supplementation).

$M S=$ materia seca; $F D N=$ fibra en detergente neutro; FDA= fibra en detergente ácido; $\pm=$ desviación típica. 
Tabla III. Composición de heces de novillos cebuínos de $380 \mathrm{~kg}$ de peso pastoreando en un SSPi basado en Leucaena leucocephala con o sin suplementación energética (Composition of feces Zebu steers grazing $380 \mathrm{~kg}$ in Leucaena leucocephala SSPi with or without energy supplementation).

\begin{tabular}{lcc}
\hline Descripción & Sin suplemento & Con suplemento \\
\hline Proteína (\%) & $11,1 \pm 0,99$ & $10,9 \pm 0,31$ \\
FDN (\%) & $61,2 \pm 2,43$ & $61,3 \pm 1,86$ \\
FDA (\%) & $41,9 \pm 0,88$ & $42,7 \pm 1,90$ \\
Cenizas (\%) & $19,0 \pm 0,97$ & $19,3 \pm 0,99$ \\
Calcio (\%) & $1,28 \pm 0,26$ & $1,27 \pm 0,09$ \\
Fósforo (\%) & $0,43 \pm 0,03$ & $0,55 \pm 0,03$ \\
\hline
\end{tabular}

FDN= Fibra en detergente neutro; FDA= Fibra en detergente ácido; $\pm=$ Desviación típica.

de MS que equivalen al 2,63 \% del peso vivo. Estos consumos son altos para los normalmente reportados en el trópico (Barahona y Sánchez, 2005) y demuestran porqué con SSPi se alcanzan mayores productividades que en sistemas tradicionales (Cuartas et al., 2014a).

La inclusión de la leucaena en mezclas de pastos mejorados modifica los patrones fermentación in vitro, aumentando la degradabilidad ruminal de la MS (Molina et al., 2013). El incremento en degradabilidad ruminal de la dieta, puede explicar el aumento de consumo de MS en bovinos que pastorean SSPi. La ingestión máxima de MS se produce cuando la digestibilidad de la dieta se encuentra entre el 66 y el $68 \%$ (Faria y Mattos 1995), aunque es posible mejorar el CMS en los animales, incrementando la digestibilidad de las fracciones potencialmente digestibles o la velocidad de paso de las fracciones no digeribles en rumen (Poppi y Norton 1995).

En este estudio, el consumo de leguminosas representó un 25,9\% del consumo total de forrajes, con lo que el consumo de gramíneas fue $74,1 \%$ (tabla II). Por su parte, Cuartas et al. (2014) reportaron una proporción de casi $30 \%$ de leguminosa y 70 \% de gramínea en la dieta de novillos de $250 \mathrm{~kg}$ sin suplementación pastoreando en un SSPi compuesto por leucaena y pasto guinea. Por su parte, Mahecha (2000), al determinar el consumo de leucaena y pasto estrella por medio de aforos antes y después del pastoreo, reportó consumos totales de 9,5 $\mathrm{kg} \mathrm{dia}^{-1}$, de los que la estrella representó el $81 \%$ y la leucaena el $19 \%$ del consumo total de forraje. En el presente estudio, la presencia de leucaena en la dieta condujo a consumos altos de proteína (tabla II), que aunque adecuados, fueron un poco más bajos que los reportados por Cuartas et al. (2014).

En la tabla III se muestra el contenido de nutrientes en las heces de los animales pastoreando en el SSPi. Elementos como el calcio se encontraron en alta concentración en las heces, siendo la excreción fecal de este elemento fue mayor a su desaparición (figura 1). Cabe resaltar que en animales en crecimiento, el $90 \%$ de calcio es utilizado en el hueso y la matriz cartilaginosa orgánica. Sin embargo, los animales evaluados en este estudio ya no tienen grandes procesos activos de crecimiento óseo (Perry, 1995), pudiendo tener excreciones de calcio mayores.
En promedio, la excreción fecal de $\mathrm{N}$ fue de $26,9 \mathrm{~kg}$ animal $^{-1}$ año $^{-1}$, correspondiendo a $0,074 \mathrm{~kg}$ de $\mathrm{N} \mathrm{dia}{ }^{-1}$. Mediante el CNCPS se estimó una excreción urinaria de $0,090 \mathrm{~kg}$ de $\mathrm{N} \mathrm{dia}^{-1}$. Esto quiere decir que los 20 animales incluidos en este estudio excretarían 2,6 t de urea año ${ }^{-1}$ en las 5,6 ha del SSPi en las que pastoreaban, lo que podría generar una producción de forraje de 5,4 $\mathrm{t}$ de MS por ha año-1 a partir de las gramíneas.

La excreción fecal de $\mathrm{N}$ encontrada en este estudio es diferente a la reportada por otros autores. Por ejemplo, para animales en sistemas intensivos de producción de carne en Europa se reportaron excreciones fecales de $40 \mathrm{~kg}$ de $\mathrm{N}$ año ${ }^{-1}$ (Van der Hoek, 2001), valores superiores a los encontrados en este estudio. En Colombia, Castro et al. (2009) reportaron que vacas Holstein pastoreando en praderas de gramíneas asociadas con leguminosas tuvieron una excreción de nitrógeno ( $\mathrm{g} \mathrm{dia}^{-1}$ ) de 216,6 y 191,16 en heces y orina, respectivamente, que equivale a excreciones fecales alrededor de $79 \mathrm{~kg}$ de N año ${ }^{-1}$. Estas diferencias obedecen a diferencias en CMS y en el contenido de $\mathrm{N}$ en la dieta de los animales.

En este estudio, la digestibilidad fue del 53,5\% para el tratamiento sin inclusión del suplemento y del $60,0 \%$ para el tratamiento con suplemento, lo que junto con el CMS, permite afirmar que los animales satisficieron sus requerimientos de los principales nutrientes. Mediante el CNCPS se estimaron consumos de $873 \mathrm{~g}_{\text {día }}{ }^{-1}$ de proteína metabolizable y de $17,8 \mathrm{Mcal}$ día $^{-1}$ de energía metabolizable, indicando que el factor limitante para alcanzar mayores ganancias diarias sería el consumo de energía. Como lo reportan diferentes estudios, el contenido de taninos de la leucaena no reduce en gran medida la degradabilidad ruminal de la proteína (Barahona et al., 2003, 2006), pero es posible esperar que una parte de ésta se proteja de forma natural al ataque por las proteasas microbianas, favoreciendo el sobrepaso de proteína al duodeno (Rodríguez et al., 2010).

Al conocer el consumo (tabla II) y la excreción fecal (tabla IV) fue posible determinar la desaparición de cada nutriente como la diferencia entre consumo y excreción fecal. Esta fracción fue denominada desapa-

Tabla IV. Excreción fecal de nutrientes de novillos cebuínos de $380 \mathrm{~kg}$ de peso pastoreando en un SSPi basado en Leucaena leucocephala con o sin suplementación energética (Fecal excretion of nutrients from $380 \mathrm{~kg}$ Zebu steers grazing in Leucaena leucocephala SSPi with or without energy supplementation).

\begin{tabular}{|c|c|c|c|}
\hline Excreción & Sin suplemento & Con suplemento & Valor $p$ \\
\hline MS kg dia-1 & $4,38 \pm 0,11$ & $3,99 \pm 0,17$ & 0,15 \\
\hline Proteína Cruda, $\mathrm{kg} \mathrm{dia}^{-1}$ & $0,49 \pm 0,04$ & $0,44 \pm 0,01$ & 0,08 \\
\hline FDN, $\mathrm{kg} \mathrm{dia}^{-1}$ & $2,68 \pm 0,14$ & $2,45 \pm 0,07$ & 0,10 \\
\hline FDA, $\mathrm{kg} \mathrm{dia}^{-1}$ & $1,84 \pm 0,07$ & $1,72 \pm 0,06$ & 0,19 \\
\hline Cenizas, $\mathrm{kg} \mathrm{dia}^{-1}$ & $0,83 \pm 0,04$ & $0,77 \pm 0,07$ & 0,20 \\
\hline Calcio, $\mathrm{kg} \mathrm{dia}^{-1}$ & $0,06 \pm 0,006$ & $0,05 \pm 0,004$ & 0,21 \\
\hline Fosforo, $\mathrm{kg} \mathrm{dia}^{-1}$ & $0,02 \pm 0,002$ & $0,02 \pm 0,002$ & 0,03 \\
\hline
\end{tabular}

MS= materia seca; FDN= fibra en detergente neutro; FDA= fibra en detergente ácido; $\pm=$ desviación típica. 


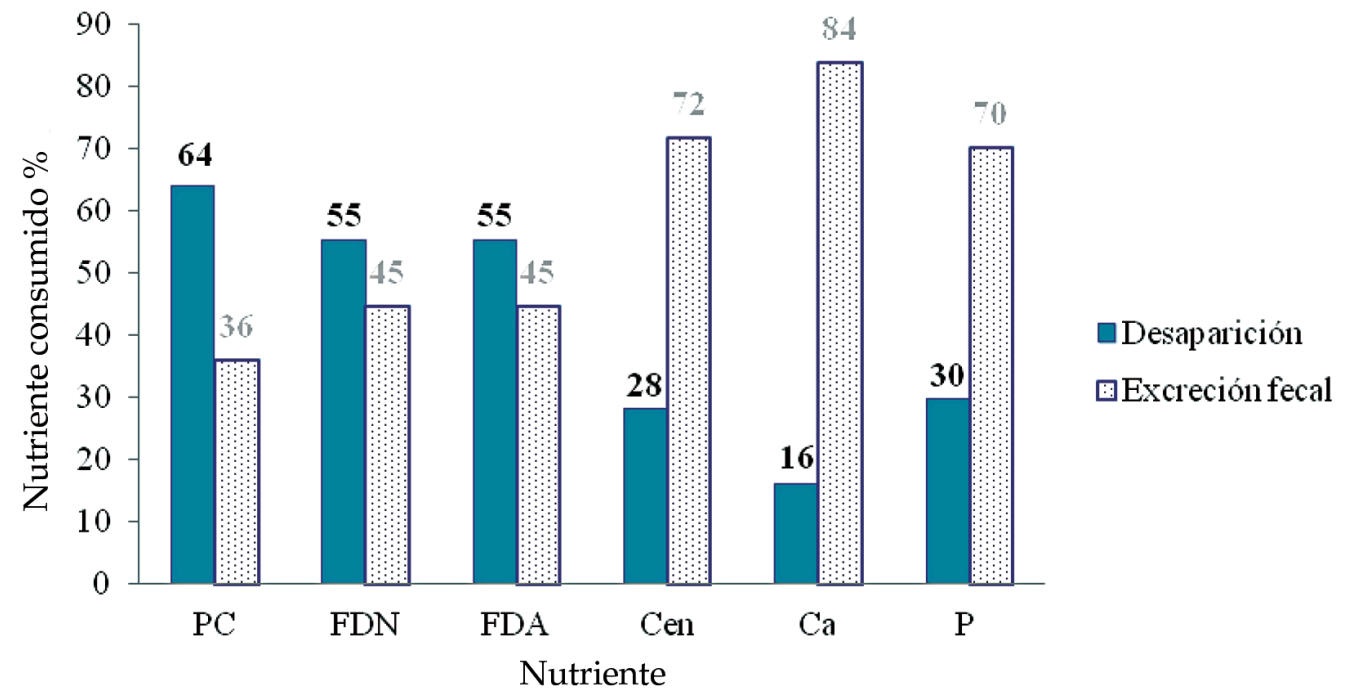

Figura 1. Desaparición y excreción fecal como porcentaje del consumo de nutrientes en novillos cebuínos de $380 \mathrm{~kg}$ de peso pastoreando en un SSPi basado en Leucaena leucocephala con o sin suplementación energética (Disappearance and fecal excretion as a percentage of consumption of nutrients in $380 \mathrm{~kg}$ Zebu steers grazing in Leucaena leucocephala SSPI with or without energy supplementation).

rición porque no se sabe cuál es su destino metabólico. En el caso de la proteína, la desaparición fue de $870 \mathrm{~g}$ dia $^{-1}$, lo que equivale al $65 \%$ de lo consumido (figura 1). Esta proteína desaparecida pudo ser aprovechada en la generación de productos o ser excretada en orina. Un comportamiento similar, en el que la desaparición fue mayor a la excreción se presentó en las fracciones de FDN y FDA. Fracciones como cenizas, Ca y $\mathrm{P}$ presentaron patrones de excreciones mayores a su desaparición.

Asumiendo los máximos valores posibles de retención de $\mathrm{N}$ en carne, en este estudio se estimaría una eficiencia de utilización de nitrógeno de alrededor de 7 \% (Gaviria et al., 2012). Estos valores son similares a los reportados para ganado de carne, los que se encuentran en promedio en 7,7 \%, lo cual representa la eficiencia promedio para convertir el nitrógeno consumido en bovinos de carne. En contraste, la eficiencia en uso de $\mathrm{N}$ reportada para cerdos es de $20,5 \%$, de $33,8 \%$ para aves de engorde y de 18,6\% para bovinos de leche (Van der Hoek, 2001).

\section{CONCLUSIONES}

El consumo de forrajes en el SSPi estudiado estuvo entre 2,46 y 2,59 \% del PV y representó cerca de $10 \mathrm{Kg}$ de MS por día en animales cebuínos de $380 \mathrm{~kg}$ de peso vivo. Estos consumos fueron adecuados para el tipo de animales del estudio, aportando una dieta de buena calidad nutricional. La excreción fecal de $\mathrm{N}$ se calculó en $29,9 \mathrm{~kg}$ animal ${ }^{-1} \mathrm{año}^{-1}$, lo que probablemente contribuye de manera importante a la producción de biomasa de las gramíneas acompañantes en el SSPi, especialmente si se considera que el número de animales por ha fue de 3,57, correspondiendo a una excreción de $\mathrm{N} \mathrm{ha}^{-1}$ año${ }^{1}$ superior a los $105 \mathrm{~kg}$.

En futuras investigaciones es necesario analizar las excreciones en orina, además de las fecales para precisar adecuadamente las proporciones de nutrientes que se metabolizan y se retienen en el animal y las que son devueltas a la pradera.

\section{AGRADECIMIENTOS}

Los autores agradecen al Ministerio de Agricultura y Desarrollo Rural por la financiación del proyecto de investigación Análisis comparativo de producción de carne en novillos producidos en un Sistema Silvopastoril Intensivo y Confinamiento dentro del cual se realizó el presente estudio. Se agradece además a Agroindustrias Santana S.A.S por proporcionar los animales con los cuales se desarrolló este estudio. Se agradece la contribución recibida por parte del equipo de trabajo en CIPAV, Udea y UNAL.

\section{BIBLIOGRAFÍA}

Akaike, H. 1974. A new look at the statistical model identification. IEEE T Automat Contr, 19: 716-723.

AOACAssociation of Official Analytical Chemists. 2005. Official Method 942.05. Determination of ash in animal feed. In official methods of analysis of AOAC international. 18 $8^{\text {th }}$ edition (Chapter 4). AOAC International. Gaithersburg, MD. USA. p. 8.

AOAC Association of Official Analytical Chemists. 2005. Official method 973.18. Fiber (Acid detergent) and lignin (H2SO4) in animal feed. In official methods of analysis of AOAC international. AOAC International. Gaithersburg, MD. USA.

AOAC Association of Official Analytical Chemists. 2005. Official Method 2002-04. Amylase-treated neutral Detergent fiber in feeds. In official methods of analysis of AOAC international. AOAC International. Gaithersburg, MD. USA

Barahona, R. y Sánchez M. 2005. Limitaciones físicas y químicas de la digestibilidad de pastos tropicales y estrategias para aumentarla. Rev Corpoica Cienc Tecnol Agropecu, 6: 69-82.

Barahona, R.; Lascano, C.; Narvaez, N.; Owen, E.; Morris, P. and Theodorou, M.K. 2003. In vitro degradability of mature and immature leaves of tropical forage legumes differing in condensed tannin and non-starch polysaccharide content and composition. J Sci Food Agric, 83: 1256-1266.

Barahona, R.; Theodorou, M.; Morris, P.; Owen, E.; Lascano, C.E. and Sánchez Pinzón, M.S. 2006. Effect of condensed tannins from tropical 
legumes on the activity of fibrolytic enzymes from the rumen fungus Neocallimastyx hurleyensis. Enzyme Microb Tech, 39: 281-288.

Castro, E.; Mojica, J.E.; León, J.; Pabón, M.; Carulla, J.E. y Cárdenas, E. 2009. Balance de nitrógeno en pastura de gramíneas y pastura de gramínea más Lotus uliginosus en la sabana de Bogotá, Colombia. Rev Corpoica Cienc Tecnol Agropec, 10: 91-101.

Córdoba, C.P.; Arango Palacio M.E.; Bolívar Vergara, DM y Barahona Rosales, R. 2008. Consumo y composición de la dieta de bovinos en pastoreo mediante el método de alcanos. Rev FacNacAgron, 62: 44-45.

Crespo, G.; Rodríguez, I.; Torres, V.; Ortíz, J. y Cabrera, G. 2000. Contribución al conocimiento del reciclaje de los nutrientes en el sistema suelo-planta-animal en Cuba. Ed Instituto de Ciencia Animal (EDICA) La Habana. Cuba. 72 pp.

Cuartas, C.A.; Naranjo, J.F.; Tarazona, A.M.; Murgueitio, E.; Chará, J.D.; Ku Vera, J.; Solorio, F.; Flores, M.; Solorio, B. and Barahona, R. 2014a. Contribution of intensive silvopastoral systems to the adaptation and mitigation of climate change. Rev Colomb Cienc Pecu, 27: 76-94

Dove, H.; Freer, M. and Foot, J.Z. 2000. The nutrition of grazing ewes during pregnancy and lactation: a comparison of alkane-based and chromium/in vitro-based estimates of herbage intake. Aust J Agr Res, 51: 765-777.

Dove, H. and Mayes, R.W. 1991. The use of plant wax alkanes as markers substances in studies of the nutrition of herbivores: a review. Aust J Agr Res, 42: 913-952.

Dove, H. and Mayes, R.W. 2006. Protocol for the analysis of n-alkanes and other plant-wax compounds and for their use as markers for quantifying the nutrient supply of large mammalian herbivores. Nat Prot, 1: 1680-1697.

Dove, H. and Moore, A.D. 1995. Using a least-squares optimization procedure to estimate diet composition based on the alkanes of plant cutilular wax. Aust J Agr Res, 46: 1535-1544.

Faria, V.P. e Mattos, W.R. 1995. Nutrição de bovinos tendo em vista performances econômicas máximas. In: A. M. Peixoto, J. C. Moura \& V. P. Faria (Eeds.). Nutrição de bovinos: conceitos básicos e aplicados. FEALQ. Piracicaba. pp. 199-222

Ferreira, L.M.; Oliván, M.; Celaya, R.; García, U.; Rodrigues, M.A.M. and Osoro, K. 2007. The use of n-alkanes to estimate diet composition of ruminants grazing on species diverse plant communities -Effect of feeding selectivity on diet composition estimates. Livest Sci, 111 : 114-123.

Gaviria, X.; Sossa, C.P.; Montoya, C.; Chara, J.; Lopera, J.J.; Córdoba, C.P. y Barahona, R. 2012. Producción de carne bovina en sistemas silvopastoriles Intensivos en el trópico bajo colombiano: Memorias de: VII Congreso latinoamericano de sistemas agroforestales para la producción animal sostenible. Belém do Pará. Brasil.

Gedir, J.V.; and Hudson, R.J. 2000. Estimating dry matter digestibility and intake in wapiti (Cervus elaphus canadensis) using the double n-alkane ratio technique. Small Ruminant Res, 36: 57-62.

Giráldez, F.J.; López, S.; Lamb, C.S. and Mayes, R.W. 2006. The use of even-chain alkanes sprayed onto herbage as rate of passage markers in goats. Livest Sci, 100: 195-202.

Herrero, M.; Grace, D.; Njuki, J.; Johnson, N.; Enahoro, D.; Silvestri, S. and Rufino, M.C. 2013. The roles of livestock in developing countries. Animal, 7: 3-18.

ICONTEC. 1973. NTC 668. Alimentos y materias primas. Determinación de los contenidos de grasa y fibra cruda. Instituto Colombiano de Normas Técnicas y Certificación. Bogotá, D.C.

ICONTEC. 1999. NTC 4657. Alimentos para animales. Determinación del contenido de nitrógeno y cálculo del contenido de proteína cruda. Método Kjeldahl. Instituto Colombiano de Normas Técnicas y Certificación. Bogotá, D.C

ICONTEC. 2001. NTC 4981. Alimentos para animales. Determinación del contenido de fosforo. Método espectrofotométrico. Instituto Colombiano de Normas Técnicas y Certificación. Bogotá, D.C.
ICONTEC. 2003. NTC 5151. Alimento para animales. Determinación de los contenidos de calcio, cobre, hierro, magnesio, manganeso, potasio, sodio y zinc. Método usando espectrometría de absorción atómica. Instituto Colombiano de Normas Técnicas y Certificación. Bogotá, D.C.

ISO. 1998. Animal feeding stuffs, animal products, and faeces or urine - ISO 9831: Determination of gross calorific value - Bomb calorimeter method. International Organization for Standardization. Geneva, Switzerland.

ISO. 1999. Animal feeding stuffs. ISO 6496: Determination of moisture and other volatile matter content. International Organization for Standardization. Geneva. Switzerland.

Littell, R.C.; Henry, P.R. and Ammerman, C.B. 1998. Statistical analysis of repeated measures data using SAS procedures. J Anim Sci, 76: 1216-1231.

Littell, R.C.; Pendergast, J. and Natarajan, R. 2000. Modelling covariance structure in the analysis of repeated measures data. Stat Med, 19: 1793-1819.

Mahecha, L.; Durán C.V.; Rosales M.; Molina C.H. y Molina E. 2000. Consumo de pasto estrella africana (Cynodon plectostachyus) y leucaena (Leucaena leucocephala) en un sistema silvopastoril. Nota de investigación. Pasturas Trop, 22: 26-30.

Mayes, R.W., Lamb, C.S. and Colgrove, P.M. 1986. The use of dosed and herbage $n$-alkanes as markers for the determination of herbage intake. J Agric Sci, 107: 161-170.

Molina, I.C.; Cantet, J.M.; Montoya, S.; Correa, G.A. y Barahona, R. 2013. Producción de metano in vitro de dos gramíneas tropicales solas y mezcladas con Leucaena leucocephala o Gliricidia sepium. Rev CES, 8: 15-31.

Murgueitio, E.; Calle, Z.; Uribe, F.; Calle, A. y Solorio, B. 2011. Native trees and shrubs for the productive rehabilitation of tropical cattle ranching lands. Forest Ecol Manag, 261: 1654-1663.

Nolan J V and Dobos R.C. 2005 . Nitrogen transanctions in ruminants. In J. Dijkstra, J.M. Forbes and J. France (eds), Quantitative aspects of ruminal digestion and metabolism. $2^{\text {nd }}$ ed. CABI Publishing. Wallingford. UK. pp. 177-206.

Perry, T.W. and Cecava, M J. 1995. Beef cattle feeding and nutrition. Cap. 3. Mineral requirements of beef cattle $2^{\text {nd }} \mathrm{ed}$. Academic Press, Inc. San Diego, California. pp. 36-51.

Poppi, D.P. and Norton, B.W. 1995. Intake of tropical legumes. In: J. P. $D^{\prime}$ Mello \& C. Devendra (Eds.). Tropical legumes in animal nutrition. CAB International. pp. 173-189.

Rodríguez, R.M.; Mota, C. and Castrillo, M. 2010. In vitro rumen fermentation of the tropical grass Pennisetum purpureum and mixtures with browse legumes: effects of tannin contents. J Anim Phys Anim Nutr, 94: 696-705.

Schwarz, G.E. 1978. Estimating the dimension of a model. Ann Stat, 6: $461-464$.

Van der Hoek, K.W. 2001. Nitrogen efficiency in agriculture in Europe and India. Optimizing nitrogen management in food and energy production and environmental protection: Proceedings of the $2^{\text {nd }}$ International nitrogen conference on science and policy. Scientific World, 1: 148-154.

Van der Hoek, K.W. 1998. Nitrogen efficiency in global animal production. In: K.W. Van der Hoek, J.W. Erisman, S. Smeulders, J.R. Wisniewski and J. Wisniewski (Edts.). Nitrogen, the Confer-N-s. Elsevier. Amsterdam. pp. 127-132.

Van Soest, P.J.; Robertson, J.B. and Lewis, B.A. 1991. Methods for dietary fiber neutral detergent fiber and nonstarch polysaccharides in relation to animal nutrition. J Dairy Sci, 74: 3583-3597.

Van Soest, P.J. 1963. Use of detergents in the analysis of fibrous feed. II. A rapid method for the determination of fiber and lignin. J Assoc Anal Chem, 46: 829 